\title{
LTE-Advanced technical aspects and standards
}

\author{
Arra Ashok; Mantha Anil Srimanth; J. Ravibabu; \\ M. Tech, Department of ECE, Sreenidhi Institute of Science and Technology \\ M. Tech, Department of ECE, Sreenidhi Institute of Science and Technology \\ M. Tech, Department of ECE, Sreenidhi Institute of Science and Technology
}

\begin{abstract}
:
Long Term Evolution (LTE) is a radio platform technology that will allow operators to achieve even higher peak throughputs than HSPA+ in higher spectrum bandwidth. LTE is part of the GSM evolutionary path for mobile broadband. The overall objective for LTE is to provide an extremely high performance radio-access technology that offers full vehicular speed mobility and that can readily coexist with HSPA and earlier networks. With the help of scalable bandwidth, operators will be able to easily migrate their networks and users from HSPA to LTE over time. The performance of radio communications depends on an antenna system. In this paper how the emerging technology is achieving high rate, high reliability, and long range communications is explained in detail. The performance of radio communications depends on an antenna system it is explained in detailed. In order for LTE-Advanced to fully utilize the wider bandwidths of up to $100 \mathrm{MHz}$, while keeping backward compatibility with LTE, a carrier aggregation scheme has been proposed. Carrier aggregation consists of grouping several LTE, so that the LTE-Advanced devices are able to use a greater amount of bandwidth. Relaying is another of the elements that is introduced in LTE-Advanced to improve the performance of LTE.
\end{abstract}

\section{Introduction}

The requirement for higher data rates are increasing highly because of the availability of smart phones and local social networks will be available at cheap rates. So wireless data networks is improvement is much needed. LTE-Advanced is an solution for the these kind of networks. LTE-Advanced is also known as the $4 \mathrm{G}$ cellular communication network. IMT-A (International Mobile Telecommunications-Advanced) has approved that this LTE-Advanced as the 4G Mobile Communication. So it is offering higher data rates with the low latency. The first workshop was held on November, $26^{\text {th }} 2007$, as an informational and educational session to inform 3GPP of relevant information related to IMT-Advanced in the ITU-R. A more detailed second workshop on IMT-Advanced and LTE-Advanced on was held on 7-8 April, 2008, attended by over 160 international participants and addressed 58 documents. During this workshop, operators' and manufacturers' views on possible requirements for LTE-Advanced as well as ideas/proposals for LTE-Advanced were exchanged and discussed. On May 27, 2008, 3GPP held a third workshop to focus specifically on the 3GPP requirements for LTE-Advanced. It will allow the cellular provider to complement their $3 \mathrm{G}$ services by offering higher data rates, lower latency and packet based network. There are significant amount of improvement that were made to be qualified as LTE-Advanced. In order to achieve this significant changes are made in both the air interface and the network architecture.

3GPP improvement is made by using the following techniques they are using of higher order of MIMO (Multi Input \& Multi Output), Heterogeneous Network, Carrier Aggregation (CA), Relaying Methods were used to improve the user experience for higher data rates while the user may have the experience for the higher data rates while the mobile unit is in motion.

Heterogeneous Network is formed by using the splitting of cell coverage area into smaller regions. they are the macro cell, microcell, picocell, femtocells, these cells were designed to get the excellent improvement for the data rates, higher coverage area which results that user is able to get the extraordinary data rates,coveragearea. In this we are able to get the higher order coverage by using the mechanism for beam forming. Macro cell which is used in the rural area within the range of $35 \mathrm{~km}$. Micro cell which is under the area range of $2 \mathrm{~km}$ to $35 \mathrm{~km}$ which is having the densely populated area. Femto cell is having the limit of few hundreds to up to $2 \mathrm{~km}$ area. Pico cell is formed in the area under the range of up to several tens to 100 meters, which is having the range will be used in the building.

Carrier Aggregation which is used in the networks to improve the quality of transmitting the signals which are combined together to transmit over a single channel which can be used for multiplexed data to be so that they can improve the bandwidth utilization up to $100 \mathrm{MHz}$.i.e, we can aggregate five carriers which are having the bandwidth which can have individually up to 20MHz.so we are able to get the bandwidth 100MHz.so this can be achieved by using the Carrier aggregation with different modes for propagation in the network which leads to the improve in the bandwidth.

Multi Input Multi Output which resulting the multiple inputs \& multiple outputs of the large amount of data which can resulting into smaller parts of data which can leads to transmission of data by using multiple antennas which can have the data to be transmitted over at a time which can leads to simultaneous data 
transmission of data to be shared by the large number of users which can resulting higher order data rates that are achieved.

Relaying is the concept of improving of higher data rates, group mobility cell edge coverage area which can be improved using this relaying technique. Increasing the spatial efficiency as a result of which is used for throughput gain which is the gain which can be cause improvement in the Signal Interference to Noise Ratio. So by using these relaying techniques the cost of the Heterogeneous network is going to reduce. The requirements for the LTE-Advanced is listed below

Table 1: LTE Requirements

\begin{tabular}{|l|l|}
\hline \multicolumn{1}{|c|}{ Technical Items } & $\begin{array}{l}\text { LTE-Advanced, IMT } \\
\text { Requirement }\end{array}$ \\
\hline $\begin{array}{l}\text { Downlink peak data } \\
\text { rates }\end{array}$ & $\begin{array}{l}1 \mathrm{Gbps} \text { (low mobility 15 } \\
\mathrm{km} / \mathrm{hr} \text { ) }\end{array}$ \\
\hline Uplink peak data rates & $\begin{array}{l}500 \mathrm{Mbps} \text { (low mobility } \\
15 \mathrm{~km} / \mathrm{hr} \text { ) }\end{array}$ \\
\hline Bandwidth & Scalable up to $100 \mathrm{MHz}$ \\
\hline User plane latency & $10 \mathrm{~ms}$ \\
\hline Control plane latency & 50 to $100 \mathrm{~ms}$ \\
\hline $\begin{array}{l}\text { Uplink peak spectral } \\
\text { efficiency }\end{array}$ & $15 \mathrm{bps} / \mathrm{Hz}$ \\
\hline $\begin{array}{l}\text { Downlink peak spectral } \\
\text { efficiency }\end{array}$ & $30 \mathrm{bps} / \mathrm{Hz}$ \\
\hline Access Scheme & OFDMA \\
\hline
\end{tabular}

So the distance between the transmitter to receiver is decreased by using the relaying mechanism so this can lead to the higher performance of data rates which is allowed to the user to be mobile for the mobility of node to achieve the peak data rates so higher data rates that can lead with the help of OFDMA(Orthogonal Frequency Division Multiple Access Scheme).It can lead the data rates upto 300Mbps on Downlink \&75 Mbps on Uplink which can lead the user to transmit the data over the long distances while the user is in mobility position it support $300 \mathrm{~km} / \mathrm{h}$ to $500 \mathrm{~km} / \mathrm{h}$ with the low latency up to $5 \mathrm{~ms}$, and 200 users per cell $5 \mathrm{MHz}$.FDD and TDD will be supported by this network and MIMO will support instead of $2 \times 2$ to $4 \times 4$ antenna's technologies. These technologies will enable to get guaranteed of Quality of Service (QoS).

\section{Literature Survey}

\subsection{TECHNOLOGICAL COMPONENTS AND CHALLENGES IN LTE-ADVANCED}

There are four components that are used to get this LTE-Advanced to become the standard for the 4G Cellular Communication. These components will result the best user experience for the higher data rates with the low latency. Heterogeneous Network, Relaying, Carrier Aggregation, MIMO support these will be used for the communication to result higher coverage area, speed and effective utilization of bandwidth, using the higher spectral efficiency to get the higher performance of the network which the user can experience the flexibility to move the data to be get the performance.

MIMO using the beamforming\&spatial multiplexing to achieve the higher data rates up to 300Mbps,aggregation of multiple LTE components that are used \& it uses the QPSK modulation which can result the higher spectral efficiency with compare to all modulation techniques, improves the cell coverage area and active bit rate.

LTE-Advanced it is aimed to support increase in the downlink spectral efficiency in $15 \mathrm{bps} / \mathrm{Hz}$ for $8 \mathrm{X} 8$ antennas and $7 \mathrm{bps} / \mathrm{Hz}$ in the uplink spectral efficiency for $4 \times 4$ antennas to be used to get the data rates. It uses the backhaul mechanism to get the spectral efficiency more. CoMP (coordinated multipoint transmission and reception) is based on cooperation between different base stations using fast backhaul network in order to significantly improve the interference situation and thus overall system performance. In CoMP, relay node is connected to a donor cell $\mathrm{Uu}$ interface. RN is usually a separate cell with own physical cell ID, synchronization channels, reference symbols, and etc. Mean while carrier aggregation increases the transmission bandwidth with multiple carriers, whether being contiguous (close to each other) or not. This way HARQ (hybrid automatic repeat request) retransmission can be performed independently per component carrier.

LTE-Advanced is the all IP based cellular networks that can offer higher user data rates and lower latency. In LTE-Advanced lower latency can be achieve by adopting terminal state of being idle or active which can significantly reduce control plane latency and signaling compare to earlier generation. Higher user data rates can be achieve by adopting several techniques including: MIMO support, Modulation techniques like OFDM, bandwidth flexibility, and the support of FDD (Frequency Division Duplex) and TDD (Time Division Duplex) modes of operation. The future techniques include carrier aggregation, high order MIMO and deployment of heterogeneous networks. Heterogeneous networks will become the key feature in terms of deployment, because it blends macro-cells to Pico-cells, micro-cells, relays and Femto-cells. With the help of HetNet, user can enjoy broadband experience in cost-effective manner. In HetNet, load balancing can be easily done with the deployment of Femto or Pico cells. 
Improving of relaying mechanism to get the each user to be close to each other i.e,transmitter to receiver distance will be reduced by using the higher level of modulating antenna's that are to be used to get the data rates that will be increasing in the network to get higher performance. Carrier aggregation improves that the data rates to be used is increased with respect to the MIMO technology. In carrier aggregation the user equipment (UE) to get the low power consumption by using this technique to be used by the User by MAC signaling from base station to user while receiving the single carrier. SFBC (Space Free Block Coding) is used for two transmitted data antenna at downlink \& FSTD (Frequency -Switched Transmit Diversity) is four transmitter antennas at downlink. In this LTE-Advanced uses $8 \mathrm{X} 8$ configuration for downlink to support the higher level of data rates. It reduces the overall traffic from macro cell and improves cell-edge coverage. In relaying, UE communicates with the network using $\mathrm{RN}$ that is wirelessly connected to donor cells using LTE radio access technologies.

The key element which results low latency this can be achieved by using the carrier aggregation which can yield that the data to be transmitted higher speed. Carrier aggregation may leads to three types of configurations they are intra band continuous carrier, intra band non continuous carrier\& inter band aggregation. This is formed to make the higher level data rates by using the fragmentation of spectral efficiency to be improved. Multiple antennas uses eight layer of transmission with the help of spatial multiplexing.

\subsection{MIMO (Multi Input Multi Output)}

By using this MIMO we are getting the higher data rates which are having that can yields up to $100 \mathrm{MHz}$ for downlink \& $50 \mathrm{MHz}$ for uplink with the bandwidth of $20 \mathrm{MHz}$ carrier for MIMO.

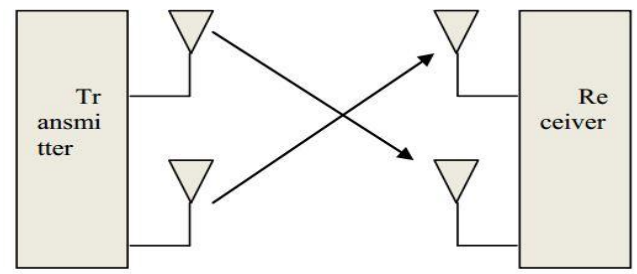

Fig. 1 Represents that 2X2 MIMO

In this figure we are having 2 antenna's for uplink and 2 antenna's for downlink that are going to transmitted over the channel this is an example of multiple input and multiple output technologies.

This can be formed improved data rates by using the higher level of MIMO technology to improve the data rates here we are using 4x4 MIMO for the uplink \& 8x8 MIMO technology for downlink data transmission which can results that can have the higher data rates to be achieved, with this MIMO technique we are having that the data rates to be achieved with the help of OFDMA i.e., Orthogonal Frequency Division Multiple Access Technique that can perform the higher data rates to be achieved. Multiple carries are combined together then they can be transmitted over channel to clubbed and they are interference can be formed carriers that can be separated in an orthogonal manner that can be formed as an OFDM signal \& gets transmitted.

Here the SC-OFDMA(Single Carrier Orthogonal Frequency Division Multiple Access) for downlink space time block code which can leads to achieve transmit diversity gain i.e., to be improved with the help of this increase in the data rates wit out sacrificing the diversity gain. The increase in the mobility for the signal to noise ratio will result can formed by channel quality.

Multiple input and Multiple Output results that can be formed to improve the higher data rate i.e., increase data rate, cell coverage area $\&$ as well as the throughput of the network is also to be improved these are the main factors that can form the higher level of data rates and requirements of the $4 \mathrm{G}$ technologies will be fulfilled by using this techniques.

SU-MIMO(Single User for Multiple Input and Multiple Output),MU-MIMO(Multiple User for Multiple Input and Multiple Output) and beam forming technique will be resulting the higher data rates can be formed and multiple signals are clubbed and transmitted. The pre-coding the matrix coding is determined by using the OFDM. Precode book is used for the $4 \mathrm{X} 4$ transmitting antennas \& spatial multiplexing is uses the $8 \mathrm{X} 8$ for downlink antenna's and $4 \mathrm{X} 4$ antenna's for uplink access.

\subsection{Heterogeneous Networks and Relaying Mechanism in LTE-Advanced}

Basic problem in the network which is relates that the data should not experience the propagation loss, so how to achieve this it is done with the help of decode and forward relay nodes that can be used it is used to increase the signal to interference noise ratio at the cell edge, cell coverage, capacity, relays nodes near the cell edge can be deployed for both coverage and cell capacity. Relaying can be fall into three categories.

Relaying can be characterized into three ways

i)Layer1 Relaying

ii)Layer2 Relaying 
iii)Layer3 Relaying

Layer1 Relaying which is consisting of "boost or repeater" mechanism .This is an Amplifier and Forward mechanism (AF) will be used. When any signal is received at the downlink on the base station are amplified and transmitted to the mobile station. In the same manner the signals were amplified and transmitted which ever received from the mobile equipment to base station is transmitted. But here we are experiencing the issue is noise is also amplified with respect to the signal whichever is received at the base station.

Layer2 Relaying is of decode and forward relaying mechanism whichever is made i.e., we can have that the signal i.e., transmitted at the downlink were first demodulated and decoded then this can be encoded and modulated again then this may be transmitted again. This can be demodulated and decoding process is performed at the radio cell station over comes the drawback which ever result the minus which has experienced that is noise is also amplified is over come here i.e., the user can be having flexibility that the noise is made separate from here and transmitted over the channel. Here we can offer the mobility control over the channel by using the Automatic Repeat Request (ARQ) is performed with respect to base station to mobile station.

Laye3 Relaying

In this type of relaying the user can experience the better type of performance.

Decode and demodulation is performed on the downlink from the base station, but performing this process performance data finally performance encoding/modulating data will be present. Layer 3 can improve the throughput by eliminating the inter-cell interference and noise finally this will be regenerating the data. this will be having the drawback is delay is caused by the user data processing modulating/demodulating and encoding/decoding process will be done.

The below figure illustrates that the heterogeneous network which is used to improve the user experience for higher data rates, higher reliability to the network.

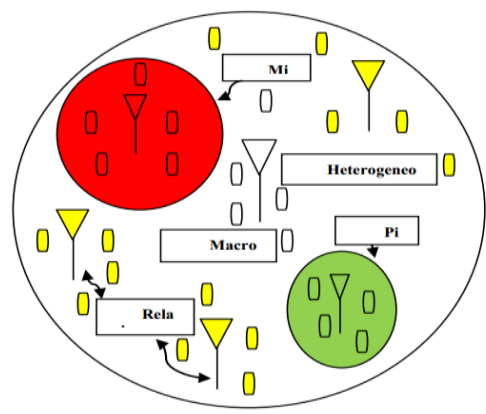

fig.2 Heterogeneous Network with relaying deployment.

Relaying is one of the most effective and enabling component of the LTE-A standard, that will improve the data rate significantly, Wireless Terminals WTs) to act as relays to other WTs in addition to transmitting their own signals. IWF (iterative-water filing) algorithm can be autonomously applied by the WTs to allocate their powers in a way that maximizes their instantaneous capacities. How to have fairness between different WTs requires the adaptation of joint scheduling and routing (JSR) algorithms. The design of distributed JSR schemes maximizes the network throughput without requiring feedback from the nodes. Fairness is a crucial aspect that must be considered in the joint design of scheduling and routing algorithms. In terminal relays, global fairness is hard to achieve because nodes can only communicate with immediate neighbor. Local fairness can be achieved by maximizing the harmonic mean rate of each wireless terminal.

\subsection{Carrier Aggregation}

It deals with the multiple carriers were associated together and transmitted over a single channel for the flexibility of bandwidth. It can receive and transmit multiple signals from user equipment. So we are having two types of carrier aggregation mechanisms were present in the network, they are contiguous carrier aggregation\& non-contiguous carrier aggregation.

In contiguous carrier aggregation we are able to transmit the signals that are arranged in continuous manner then they are transmitted over the channel. They are arranged in multiple carriers components were adjacent to each other.

In this carrier aggregation we are having different types of bandwidth of $1.4,3,5,10,15,20 \mathrm{MHz}$ bandwidth maximum of five carriers were aggregated i.e., arranged in any of the type of techniques were used, so we are able to get the bandwidth of maximum $100 \mathrm{MHz}$.

Number of carriers were aggregated is different from the Downlink \&Uplink. Here we are offering that the maximum no of carriers were used in the Uplink is less than the Downlink, the individual components used is different bandwidths. 


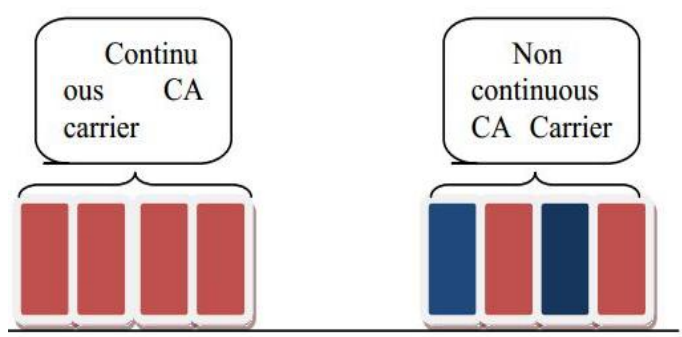

Fig. 3 Continuous CA and Non continuous CA.

Noncontiguous CA uses components that are non-adjacent and spread out in the frequency band. Continuous CA can be achieving with the help of single fast Fourier transform (FFT) using a single radio frequency, it can also provide backward compatibility with previous releases of LTE. Carrier aggregation can also resolve handover problems.

For any wireless system is to provide the fast and seamless handover from the cell to cell.. Handover related information is exchanged between the UE and target eNB via the new radio path after the UE context is fetched by the target eNB from the source eNB (thus, the usage of the term 'forward'). In non-continuous CA, component carriers required a guard between to make the interferences between the frequencies to be negligible. It is necessary for both the continuous and non-continuous CA to setup a guard-band to minimize intra and inter band interferences.

Latency in IMT-Advanced systems requires advanced handover schemes called like EBB (entry before break). To support both the current and legacy technology of 802.16 three deployments scenarios were discussed green field, mixed deployment with carrier reuse, and mixed deployment with carrier overlay. In mixed deployment the frame structure is partitioned into LZone (legacy) and MZone (current) in time-division fashion. Advanced handover schemes suggested as follows: Seamless handover in which the MS is able to exchange (send or receive) data packet data units (PDUs) with the target BS before initiating a network re-entry control message transaction, In EBB, An MS (mobile station) disconnects from the serving BS before executing handover at the target BS (base station) in BBE handover. This implies that data interruption due to handover occurs right after handover execution. When performing EBB, based on its capability, the MS performs network re-entry at the target BS during the negotiated network re-entry procedure intervals, while maintaining communications with the serving BS for data exchange up to the point of completion of network re-entry at the target BS. Thus, interruption occurs only during the negotiated network re-entry procedure intervals at the target $\mathrm{BS}$, which can be finely scheduled to minimize the total interruption time during handover. In Legacy support handover, MSs with multicarrier capability can perform seamless handover by keeping connection with the serving BS and performing handover (i.e., network re-entry) at the target BS in a parallel manner. Hence, this feature and capability allow zero tolerant data interruption time during handover.

\subsection{Comparison between Wimax and LTE}

WiMAX stands for "World Interoperability for Microwave Access", it is based on IEEE 802.16 and is similar to Wi-Fi/IEEE 802.11 networks with the coverage and QoS (quality of service) of cellular networks. It operates on both the licensed and un-licensed band compare to Wi-Fi, which only operates in ISM band, thus reducing interferences problem. It can provide broadband services up to 30 miles using fixed stations and 3-10 miles on using mobile stations. LTE and WiMAX are parallel wireless broadband technologies.

Wi-Max we can use it in the licensed and unlicensed versions. I-Max is providing that the accessing schemes were SC-FDMA for uplink and OFDMA for downlink channel we are getting better efficiency with these techniques were able to achieve the desired data rates. Band of operation is $2.3 \mathrm{GHz}$ to $5 \mathrm{GHz}$. It has the cell radius up to $2-5 \mathrm{~km}$ range. It uses the MIMO by using the $2 \times 2$ MIMO techniques were used. High speed data services availability on mobile devices is only possible with the deployments of WiMAX LTE. These two key technologies guarantee data rates at cheaper cost to the user. WiMAX is basically designed to provide the broadband wireless connectivity to fixed and nomadic users for the last mile. The coverage can go up to $50 \mathrm{Km}$, allowing user to get broadband connectivity in NLOS conditions. It uses OFDMA as an access technique that allows data rates up to $75 \mathrm{Mbps}$. It is an IEEE standard 802.16e. LTE is evolved for UMTS cellular technology. It also uses OFDM technique and supports different carrier frequency bandwidths in both FDD and TDD modes. It is a 2 node architecture means only 2 nodes are involved between the user equipment and the core network. Here is an illustration of data rates availability. 


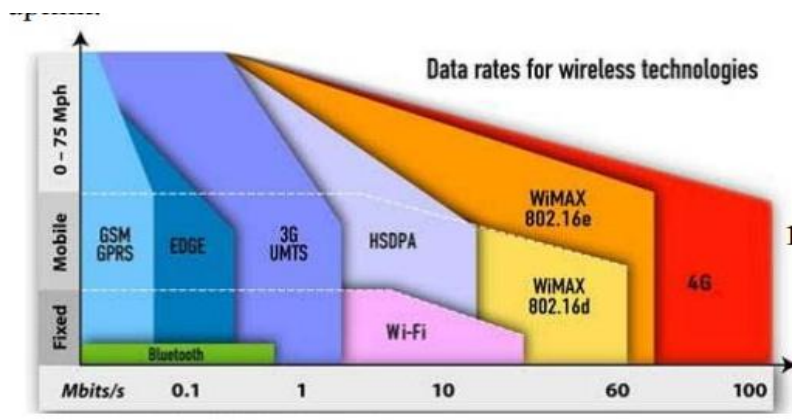

Fig.3 Data rates for wireless technology evolution.

\subsubsection{Radio Access for Interference.}

In Wi-Max we are having that single antenna which is used for the transmission of Uplink which can be used as the MIMO technique, but in LTE-Advanced we are providing that more number of antenna's for the data transmission.

\subsubsection{Protocol Architecture}

Wi-Max is having that two level of layers were present they are Physical \& MAC(Medium Access Control).MAC is again divided into 3 layers\&WiMAX but it uses first three layers of OSI model. The radio link specific protocols, including radio link control (RLC) and medium access control (MAC) protocols are terminated in the eNB.

\section{LTE-Advanced/4G Benefits\& Deployment}

4G technologies will be higher data rates in less cost i.e., cost effective nature. It can increase the coverage area, higher data rates\& cell-edge performance. So it is all-IP based network, it will be providing better Quality of Service(QoS).It is having the main benefit of spectral efficiency by using the techniques like carrier aggregation significantly increase the data rates like higher data rates which can have the spectral efficiency will be more. LTE-Advanced will be providing the world wide functionality like roaming effects, service of compatibility, interworking with the other radio issues will be present. LTE-Advanced will be compatible with the older LTE devices can be operated. increased peak data rate, DL 3 Gbps, UL 1.5 Gbps higher spectral efficiency, from a maximum of $16 \mathrm{bps} / \mathrm{Hz}$ in R8 to $30 \mathrm{bps} / \mathrm{Hz}$ in R10 ,increased number of simultaneously active subscribers improved performance at cell edges, e.g. for DL $2 \times 2$ MIMO at least 2.40 $\mathrm{bps} / \mathrm{Hz} / \mathrm{cell}$.

\section{Research Challenges}

The use of wider bandwidths, multiple spectrum bands, and spectrum sharing introduces new challenges in terms of transceiver, signal processing, resource management, and error control mechanism design, among others

\subsection{Transceiver design for carrier aggregation}

The design of wideband transceivers will be affected by several factors such as the following.

- Frequency-dependent path loss: As higher frequencies are used, the path loss increases nonlinearly.

- Doppler frequency and spectrum: At higher frequencies, the Doppler effects affect the signals more severely, which would require faster adaptation algorithms increasing the overhead.

- Effective noise power: As the bandwidth increases, the effective noise increases as well.

- Receiver input signal: Using a wider bandwidth translates into receiving more undesired signals from other services (e.g. broadcast and radar signals). So, issues such as image rejection, reciprocal mixing have to be considered.

- Nonlinearities in analogue receiver components: Distortion and inter modulation create additional signals under overload conditions, which can affect the demodulation process.

\subsubsection{Increased FFT size}

LTE utilizes up to $20 \mathrm{MHz}$ bandwidth, for which it requires a 2048 point FFT. In the case of LTEAdvanced, a bandwidth of $100 \mathrm{MHz}$ requires an FFT of increased size. If we follow the trend in LTE of FFT size versus bandwidth, for $100 \mathrm{MHz}$, an FFT size of 10240 would be needed. This will directly affect the memory size, and the base-band processing power requirement. 


\subsubsection{Resource management}

The option of using more than one spectrum band (either dedicated or shared) is immediately followed by the decision of how many bands and which bands should be used in order to satisfy the different constraints and requirements (delay, jitter, rate, interference, power consumption, mobility, reliability, subscription plan, cove-rage path loss, fading, Doppler effect, etc.). As explored in, the lower-frequency bands are better suited for longer-range, higher-mobility and lower-capacity systems, while higher-frequency bands are better suited for shorter range, lower mobility, and higher capacity systems. This decision should also take into account the capabilities of the UE: multiple band support, minimum and maximum distance between component carriers, and minimum and maximum number of frequency bands that can aggregate.

\subsubsection{Retransmission control}

LTE uses a combination of ARQ (at the RLC layer) and hybrid ARQ (at the MAC layer) in order to achieve the low error probability required to achieve $100 \mathrm{Mbps}$. Both methods complement each other to avoid excessive over-head while achieving high throughput, specially taking into account the relation between the error probability and throughput in TCP.

In LTE-Advanced, shorter delays are expected in LTE-Advanced. In order to achieve these high data rates and small delay, the interaction of ARQ and hybridARQ must be revisited to examine their current maximum performance and analyze any adaptations required to achieve the expected data rates and delay targets.

\subsection{Physical Size limitations at the UE for MIMO}

The main challenge regarding the support for four antennas at the UE is related to the physical space limitations. As is well known, a reduced spatial separation between the antennas of the same array can significantly decrease the achievable MIMO gain, and this fact becomes a problem when trying to accommodate a large number of antennas with adequate spacing in a handset device.

\subsubsection{Feedback design}

The uplink feedback channel is a bottleneck for the system performance in an FDD system. Many of the new features included in LTE-Advanced require an increased quantity of channel information, and very efficient feed-back schemes are needed in terms of lower resource usage and finer granularity of the CSI knowledge at the eNB.

\subsection{Location and type of relay}

- Coverage provided by other RNs and eNBs.

- RN "type" and "add-ons" (MIMO, carrier aggregation, CoMP).

- Type of cooperation between RNs

\subsubsection{User equipment as relay}

The possibility of using UEs as RNs is not one of the main approaches followed by LTE-Advanced to improve coverage and performance. Efficient procedures for searching UEs in emergency situations, routing, and selection of the most appropriate UE relay would be needed.

\section{Conclusion\& Open Issues}

The paper provides an overview of LTE-Advanced and its technological components that had been considered for 4G cellular system. Carrier Aggregation and multiple antennas techniques are based on LTE Rel8, but coordinated multi-point transmission and reception (CoMP), relaying and Het Net are still open issues. These components can surely fulfill the requirement of $1 \mathrm{Gbps}$ downlink and 500Mbps uplink data rates. Celledge performance can be tremendously improved with the deployment of low power relay nodes within a cell network. Carrier aggregation can make use of spectrum very efficiently by aggregating multiple carriers with the help of Orthogonal Division Multiple access scheme.

\section{References}

[1] Ian F. Akyildiz, David M. Gutierrez-Estevez, Elias Chavarria Reyes "The evolution to 4G cellular systems: LTE-Advanced" Physical Communication 2010

[2] Rahat Ali Khan, Asad Ali Shaikh "LTE Advanced: Necessities And Technological Challenges For $4^{\text {th }}$ Generation Mobile Network" International Journal Of Engineering And Technology Volume 2 No. 8, August, 2012.

[3] Edward Kasem, Jan Prokopec "The Evolution Of Lte To Lte-Advanced And The Corresponding Changes In The Uplink Reference Signals" Elektrorevue Issn 1213-1539 Vol. 3, No. 2, June 2012.

[4] Jolly Parikh, Anuradha Basu "Lte-Advanced: The 4g Mobile Broadband Technology" International Journal Of Computer Applications (0975 - 8887) Volume 13- No.5, January 2011 
[5] Prakash Bhat, Satoshi Nagata, Thomas Derham, Guangyi Liu And Xiaodong Shen, "Lte-Advanced: An Operator Perspective" IEEE Communications Magazine February 2012.

[6] Stefan Parkvall, Erik Dahlman, George Jöngren, Sara Landström And Lars Lindbom "Heterogeneous Network Deployments In Lte" Ericssonreview - 22011.

[7] Rizwan Ghaffar And Raymond Knopp "Interference-Aware Receiver Structure For Multiuser Mimo And Lte" Ghaffar And Knopp Eurasip Journal On Wireless Communications And Networking 2011.

[8] Helmut Bölcskei, Eth Zurich "Mimo-Ofdm Wireless Systems: Basics, Perspectives, And Challenges" IEEE Wireless Communications August 2006.

[9] Jagadeesh Babu. K, Sri Rama Krishna. K, Pratap Reddy. L “A Review On The Design Of Mimo Antennas For Upcoming 4g Communications" International Journal Of Applied Engineering Research, Dindigul Volume 1, No 4, 2011.

[10] Guangxiang Yuan, Xiang Zhang And Wenbo Wang, Yang Yang "Carrier Aggregation For Lte-Advanced Mobile Communication Systems" IEEE Communications Magazine February 2010.

[11] A. Z. Yonis, M. F. L. Abdullah and M. F. Ghanim "Effective Carrier Aggregation On The Lte-Advanced Systems" International Journal Of Advanced Science And Technology Vol. 41, April, 2012.

[12] Aimi S. A. Ghafar, N. Satiman, N. Fisal, Siti M. M. Maharum, Faiz A. Saparudin, Rozeha A. Rashid "Relay Architectures For LteAdvanced Network".

[13] Yang Yang, Honglin Hu And Jing Xu, Guoqiang Mao "Relay Technologies For Wimax And Lte-Advanced Mobile Systems" IEEE Communications Magazine October 2009.

[14] Saleh Ali Alomari A, Wafaa A.H Ali Alsalihy B, Mohammad Azmi Al-Madi C, Putra Sumari C “A New Fair Load Balancing Method For Multiple Component Carriers In 3gpp Lte-Advanced System" 2nd World Conference On Innovation And Computer Sciences - 2012 Awerprocedia Information Technology \& Computer Science (2012) 396-404.

[15] Oh-Soon Shin, Salah Eddine Elayoubi Yeon Kyoon Jeong And Yoan Shin "Advanced Technologies For Lte Advanced" Eurasip Journal On Wireless Communications And Networking 2013, 2013:25.

[16] Takehiro Nakamura, Sadayuki Abeta, Mikio Iwamura, Tetsushi Abe And Motohiro Tanno "Overview Of Lte Advanced And Standardization Trends" NTT Docomo Technical Journal Vol 12 No 2.

[17] Jolly Parikh, Anuradha Basu "LTE Advanced: The 4G Mobile Broadband Technology" International Journal of Computer Applications (0975 - 8887) Volume 13- No.5, January 2011.

[18] Hua Wang, Claudio Rosa, and Klaus Pedersen "Performance Analysis of Downlink Inter-band Carrier Aggregation in LTEAdvanced",

[19] ZuhanisMansor, EvangelosMellios, Geoffrey Hilton, Joe McGeehan, and Andrew Nix "MIMO Exploitation of 3D Multipath Statistics in a Heterogeneous LTE-Advanced Network" International Journal of Antennas and Propagation Volume 2013, Article ID 931527, 15 pages.

[20] Juho Lee, Jin-Kyu Han, and Jianzhong (Charlie) Zhang "MIMO Technologies in 3GPP LTE and LTE-Advanced" EURASIP Journal on Wireless Communications and NetworkingVolume 2009, Article ID 302092, 10 pages.

[21] Aws Zuheer Yonis and Mohammad Faiz Liew Abdullah "Wider Bandwidth of non-Contiguous Component Carriers in LTEAdvanced" International Journal of Future Generation Communication and Networking Vol. 6, No. 2 , April, 2013.

[22] Stefan Parkvall, Erik Dahlman, Anders Furuskär, Ylva Jading, Magnus Olsson, Stefan Wanstedt, Kambiz Zangi "LTE-Advanced Evolving LTE towards IMT-Advanced" IEEE-2008.

[23] A. Z. Yonis and M. F. L. Abdullah "Sophistication Techniques of Fourth Generations in Neoteric Mobile LTE and LTE-Advanced" International Journal of Cyber-Security and Digital Forensics (IJCSDF) 1(3): 167-176, 2012 (ISSN: 2305-0012).

[24] DavidMartn-Sacrist an, Jose F.Monserrat, Jorge Cabrejas-Penuelas, Daniel Calabuig, Salvador Garrigas, and Narcs Cardona "On the way towards Fourth Generation Mobile: 3GPP LTE and LTE-Advanced" EURASIP Journal on Wireless Communications and Networking Volume 2009, Article ID 354089, 10 pages.

[25] Puneet Sharma Rakesh Gill "LTE and LTE Advanced" PARIPEX - INDIAN JOURNAL OF RESEARCH Volume: 2 | Issue : 7 | July 2013.

[26] A. Z. Yonis, M. F. L. Abdullah and M. F. Ghanim "Design and Implementation of Intra band Contiguous Component Carriers on LTE-A" International Journal of Computer Applications (0975 - 8887) Volume 41- No.14, March 2012.

[27] Omer Bulakci "On Backhauling of Relay Enhanced Networks in LTE-Advanced" Licentiate Seminar, Department of Communications and Networking, Aalto University, 2010.

[28] Thomas Delbecke, Ranjith Kumar Duraisamy, Houda Rizqi, Xing Ge Xu "LTE-Advanced - Release 10 Relaying”.

[29] Muhammad Mustaqim, Khalid Khan and Muhammad Usman "LTE-Advanced: Requirements and Technical Challenges for 4G Cellular Network" Journal of Emerging Trends in Computing and Information Sciences VOL. 3, NO. 5, May 2012.

[30] Jeng-Yueng Chen and Yi-Ting Mai Chun-Chuan Yang "Handover Enhancement in LTE-Advanced Relay Networks" IEEE-2012.

[31] Amit Kumar, Dr. Yunfei Liu and Amit Wason. "LTE-Advanced: The Roadmap To 4G Mobile Wireless Networks" Global Journal of Computer Science and Technology Vol. 10 Issue 4 Ver. 1.0 June 2010. 